\title{
Correction to: Inhibited vertical mixing and seasonal persistence of a thin cyanobacterial layer in a stratified lake
}

\author{
Bieito Fernández Castro ${ }^{1,2}$ [D $\cdot$ Oscar Sepúlveda Steiner ${ }^{1}\left(\mathbb{D} \cdot\right.$ Deborah Knapp $^{3}$ (D) Thomas Posch $^{3}$ (D) \\ Damien Bouffard ${ }^{4}\left[\right.$ Alfred Wüest $^{1,4}$
}

Published online: 15 April 2021

(c) Springer Nature Switzerland AG 2021

\section{Correction to: Aquatic Sciences (2021) 83:38 \\ https://doi.org/10.1007/s00027-021-00785-9}

Figures 2 and 7 in the original published manuscript contained errors. Those are amended here.

\section{Bieito Fernández Castro}

bieito.fernandezcastro@epfl.ch;

b.fernandez-castro@soton.ac.uk

1 Physics of Aquatic Systems Laboratory, Margaretha Kamprad Chair, Institute of Environmental Engineering, École Polytechnique Fédérale de Lausanne, Lausanne, Switzerland

2 Present Address: Ocean and Earth Science, University of Southampton, Southampton, UK

3 Limnological Station, Department of Plant and Microbial Biology, University of Zurich, Seestrasse 187, Kilchberg, Zurich, Switzerland

4 Eawag, Surface Waters-Research and Management, Swiss Federal Institute of Aquatic Science and Technology,

Kastanienbaum, Switzerland 

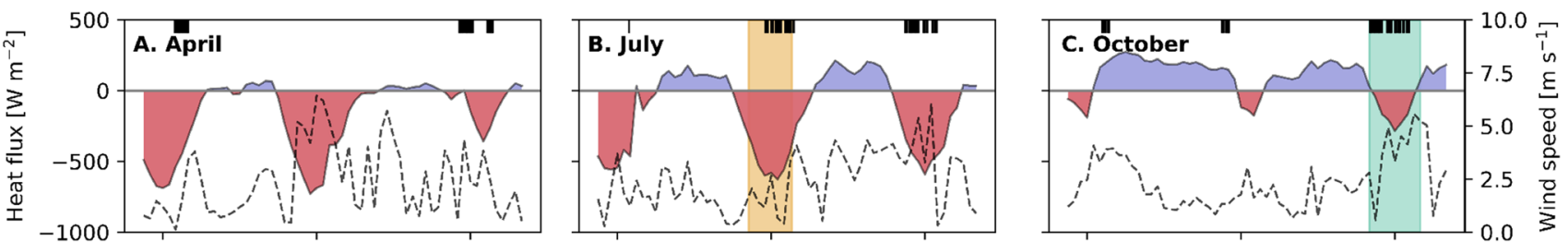

12pm-24-Apr 12pm-25-Apr 12pm-26-Apr 12pm-17Jul 12pm-18Jul 12pm-19Jul
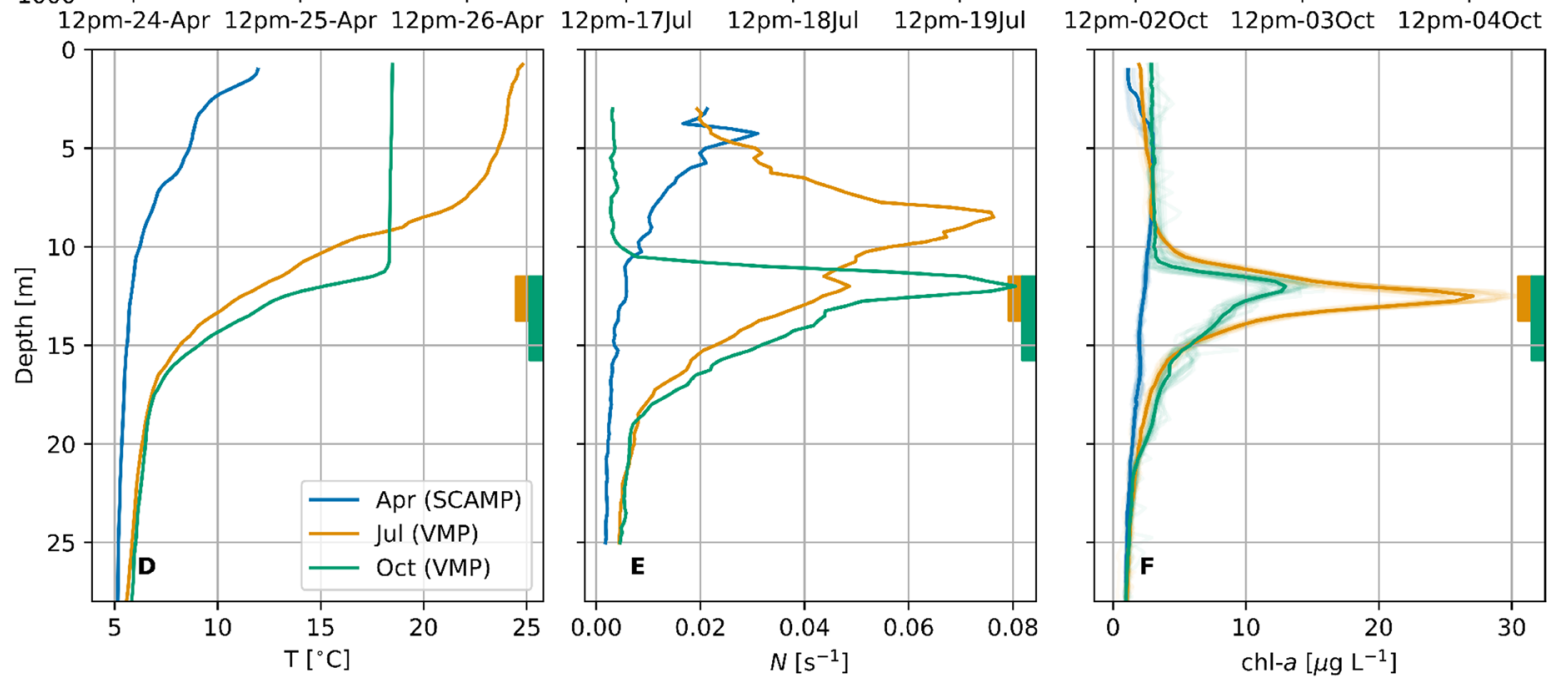

Fig. 2 Hourly meteorological conditions (net heat flux, blue-red filling; wind speed, dashed black) derived from the COSMO model during the 3 microstructure and mooring samplings in 2018: a 24-26 April 2018, b 17-19 July 2018, and c 2-4 October 2018. Median profiles of $\mathbf{d}$ temperature, $\mathbf{e}$ buoyancy frequency and $\mathbf{f}$ chl- $a$ fluorescence derived from the microstructure profiles in st1 for April 2018 (blue, SCAMP profiler), July 2018 (orange, VMP profiler) and October 2018 (green, VMP profiler). In a-c, microstructure profiles are indi-

cated with black ticks, and the duration of mooring deployments is indicated with color filling. In $\mathbf{c}-\mathbf{f}$, the depth range of the $P$. rubescens thin layer, defined as the range where chl- $a$ is larger than an e-folding fraction of the maximum value (chl- $a>\max (\operatorname{chl}-a) / e$ ), is indicated by filled rectangles for July (orange) and October (green). Individual profiles for chl- $a$ are shown in $\mathbf{f}$ as semi-transparent lines. Individual profiles were projected onto isothermal coordinates to remove the effect of internal wave motions (color figure online) 


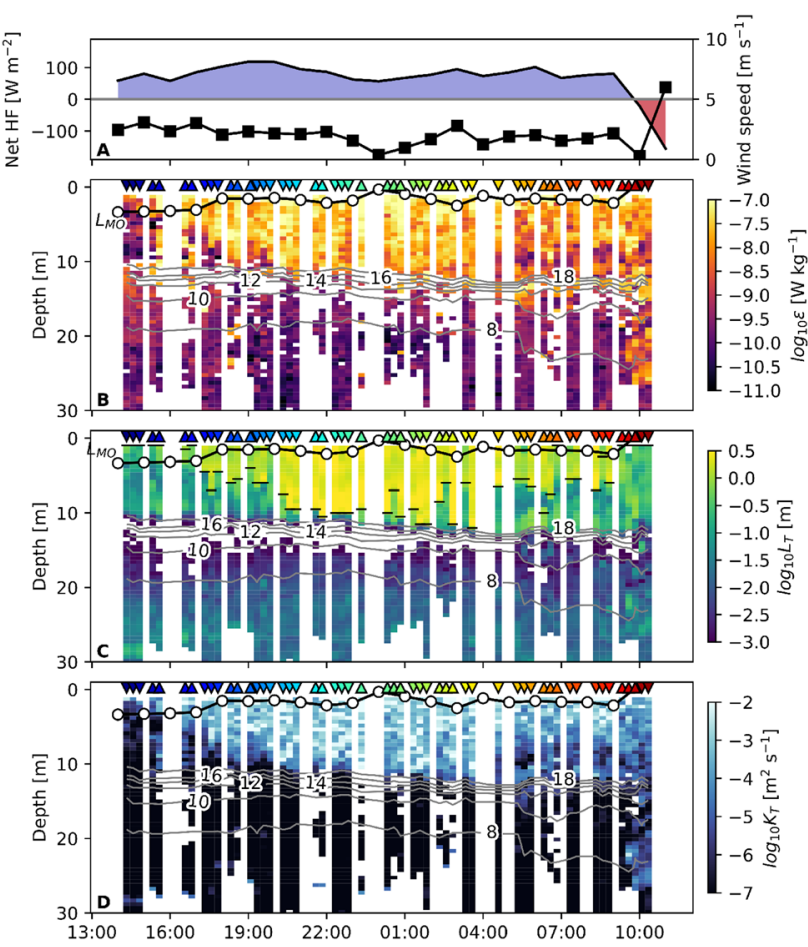

Fig. 7 Microstructure measurements collected with a microCTD profiler during the dedicated nighttime sampling on 24-25 September 2019 at st0. a Hourly surface net heat flux (colors) and wind speed (black) derived from COSMO model, b TKE dissipation rate $(\varepsilon)$, $\mathbf{c}$ Thorpe scale $\left(L_{T}\right)$, and $\mathbf{d}$ heat diffusivity $\left(K_{T}\right)$. Temperature contours every $2{ }^{\circ} \mathrm{C}$ and hourly values of the Monin-Obukhov length-scale ( $L_{M O}$, black line with dots) are plotted in b-d. Upward and downward looking triangles indicate ascending and descending profiling, respectively. The color scale of the triangles represents the sampling time. Surface mixing-layer depth (defined as $L_{T}>1 \mathrm{~m}$ ) is indicated with horizontal black markers in c. Time in the $\mathrm{x}$-axis UTC time (local time was UTC+2) (color figure online) 